\title{
Specialty distribution of physician assistants and nurse practitioners in North Carolina
}

\author{
Erin P. Fraher, PhD, MPP; Perri Morgan, PhD, PA-C; Anna Johnson, PhD, MSPH
}

\begin{abstract}
Physician workforce projections often include scenarios that forecast physician shortages under different assumptions about the deployment of physician assistants (PAs) and nurse practitioners (NPs). These scenarios generally assume that PAs and NPs are an interchangeable resource and that their specialty distributions do not change over time. This study investigated changes in PA and NP specialty distribution in North Carolina between 1997 and 2013. The data show that over the study period, PAs and NPs practiced in a wide range of specialties, but each profession had a specific pattern. The proportion of PAs-but not NPs-reporting practice in primary care dropped significantly. PAs were more likely than NPs to report practice in urgent care, emergency medicine, and surgical subspecialties. Physician workforce models need to account for the different and changing specialization trends of NPs and PAs.
\end{abstract}

Keywords: physician assistant, nurse practitioner, specialty, workforce, primary care, specialty trends

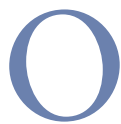
ne of the most pressing questions facing state and national policymakers is whether the increased demand for healthcare services due to an aging population, expanded health insurance coverage, and increased burden of chronic disease will result in a physician shortage. The Association of American Medical Colleges (AAMC) forecasts a shortage of 46,100 to 90,400 physicians by 2025 and the Health Resources and Services Administration (HRSA) estimates a shortfall of between 6,400 and 20,400 primary care physicians by $2020 .{ }^{1,2}$ Other workforce models suggest that the overall supply of physicians will be adequate and that the bigger issue facing the healthcare system will be the persistent maldistribution of physicians by geography and specialty.,

Erin P. Fraher is an assistant professor in the departments of family medicine and surgery at the University of North Carolina at Chapel Hill's School of Medicine. Perri Morgan is a professor in the Department of Community and Family Medicine at Duke University School of Medicine in Durham, N.C. Anna Johnson is a consultant in the Department of Epidemiology at the University of North Carolina at Chapel Hill. The authors have disclosed no potential conflicts of interest, financial or otherwise. DOI: 10.1097/01.JAA.0000481402.98969.07
Workforce models reach different conclusions about the future for many reasons, including the use of different data sources and diverging assumptions about the factors that will drive future demand and supply. To account for uncertainty caused by rapid changes in the healthcare system, models often include scenarios with a range estimates about how a particular variable (for example, hours worked, retirement rates, or population growth) will affect supply or demand. Increasingly, workforce models include scenarios about how the deployment of physician assistants (PAs) and nurse practitioners (NPs) might affect the magnitude of physician shortages under different assumptions. These scenarios are based on studies indicating that physician shortages could be partially mitigated by the increased use of interprofessional teams, including PAs and NPs, and by new models of care that make maximal use of other healthcare professionals. ${ }^{5,6}$ Dill and colleagues found broad acceptance of PAs and NPs by patients, with $23 \%$ of patients reporting that, should they need a new primary care provider, they would choose a PA or NP over a physician.?

PAs and NPs are a flexible workforce, able to fill a range of emerging healthcare needs. This flexibility occurs as new graduates sort into different specialties after completing training and when practicing PAs and NPs switch specialties during the course of their careers. ${ }^{8}$ PAs and NPs are present in all areas of healthcare in the United States. ${ }^{9}, 10$ Although PAs and NPs are similar in some ways, they differ with respect to their history, legal scope of practice, and training emphases. ${ }^{11-13}$ Due to these differences, they fill different niches in the healthcare workforce. For example, NPs are more prevalent in primary care, and PAs are more prevalent in procedural specialties such as surgery and emergency medicine, although both professions can and do practice across specialties. ${ }^{14,15}$ In 20 states, NPs may practice independently; PAs require a supervising physician in all states. ${ }^{16}$ Specialty distributions of PAs and NPs have changed over time, with the proportion of PAs and, to a lesser extent, NPs in primary care decreasing in recent years. ${ }^{17}$

Despite growing evidence that PAs and NPs practice in different specialties and evidence that PAs and NPs change specialties over time, most workforce projection models assume a constant distribution of PAs and NPs 


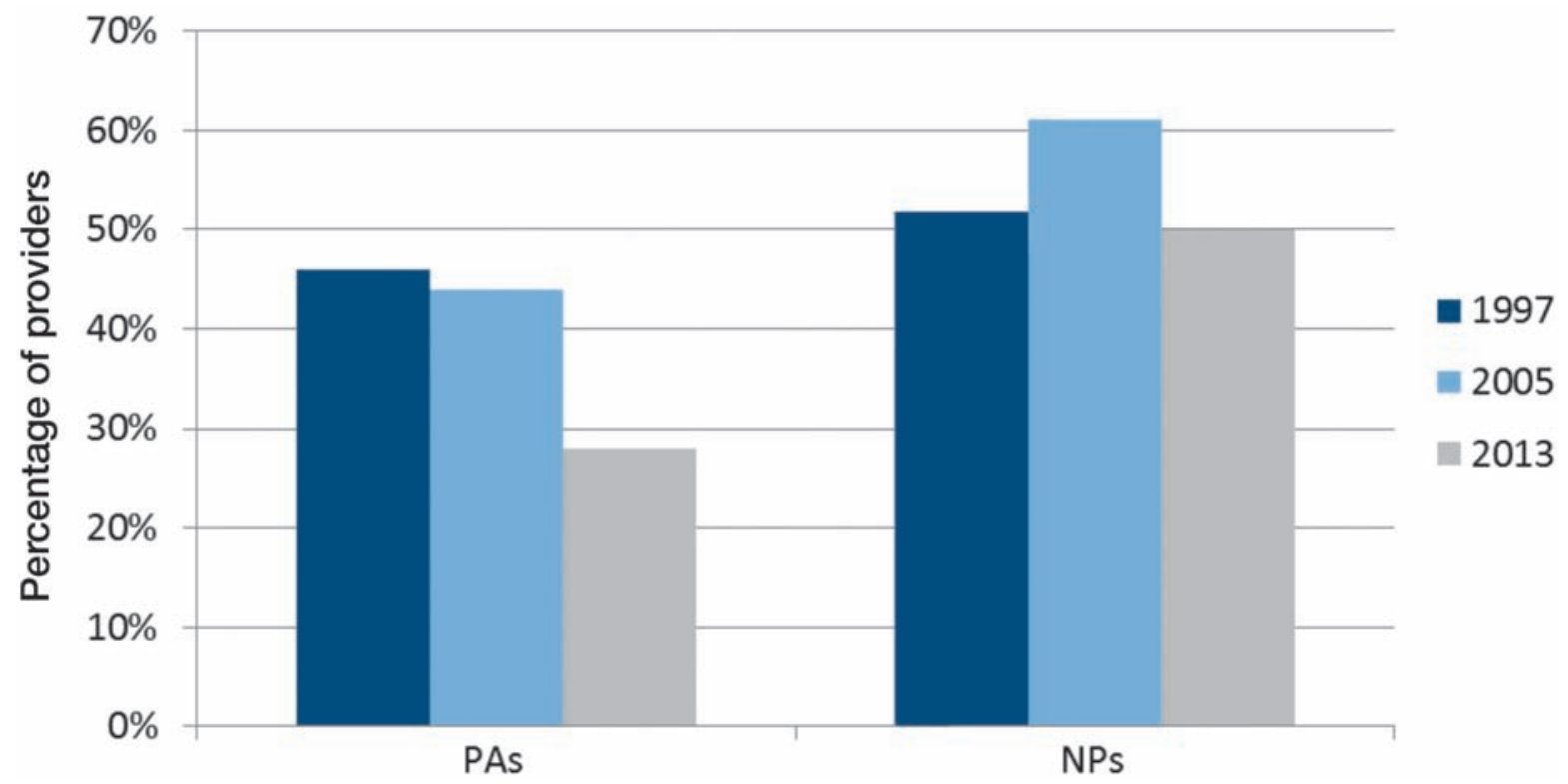

FIGURE 1. Percentage of active PAs and NPs who reported working in primary care in North Carolina in 1997, 2005 , and 2013. Primary care is defined as family medicine, general internal medicine, general pediatrics (including adolescent medicine), and general geriatrics.

between different specialties into the future. They also assume that PAs and NPs are an interchangeable resource (that is, that they work in the same specialties). This study investigated changes in the number and specialty distribution of PAs and NPs in North Carolina between 1997 and 2013. North Carolina houses a unique longitudinal data set that captures all licensed PAs and NPs in the state, making it an ideal state in which to track and compare PA and NP specialty trends.

\section{METHODS}

The source of data for this study was the North Carolina Health Professions Data System (HPDS), which is maintained at the Cecil G. Sheps Center for Health Services Research at the University of North Carolina at Chapel Hill. The HPDS contains more than 30 years of data on 19 health professions from 11 different licensure boards, and is the oldest continuous state-level health workforce data system in the country. Data on the PA and NP workforce were derived from annual licensure files from the North Carolina Board of Medicine and North Carolina Board of Nursing, respectively. Because licensure is required to practice in the state and PAs and NPs are required to reregister annually, licensure data provide a complete picture of the PA and NP workforce in the state as of October 31 of each year. The analysis data set included only PAs and NPs in active practice in North Carolina and excluded healthcare professionals who held a North Carolina license but practiced outside the state or were not in active practice. This study used self-reported specialty data for PAs and NPs actively practicing in North Carolina in 1997, 2005, and 2013.

The definition of primary care includes family medicine, general internal medicine, general pediatrics (including adolescent medicine), and general geriatrics. All other specialties were classified as specialty care. Because general pediatrics has historically been a common area of practice for NPs, we analyzed this group separately in some of the analyses. In these subanalyses, we use a "general primary care" category that includes only family medicine, general internal medicine, and general geriatrics. We used descriptive statistics to analyze specialty distributions of PAs and NPs.

\section{RESULTS}

- Overall PA and NP workforce grew rapidly. In 2013, the number of PAs and NPs in the state was roughly equal, with 4,606 PAs and 4,817 NPs in active practice. Mirroring national trends, the PA workforce increased by $259 \%$ and the NP workforce by 335\% between 1997 and 2013. The PA and NP workforces grew much more rapidly than the physician workforce, which increased $56 \%$ over the same time period. In 1997, North Carolina had 8.6 PAs and 7.7 NPs per 100 physicians, but by 2013 these ratios had more than doubled to 20.5 PAs and 21.5 NPs per 100 physicians. PA, NP, and physician growth outpaced North Carolina's population growth, which only increased by 29\% between 1997 and 2013.

- PA and NP specialty distribution changed over time. NPs were more likely to report practicing in primary care than PAs in all 3 years. In 1997, 52\% of NPs and $46 \%$ of PAs 
were in primary care. By 2013, the percent of the PA workforce reporting practice in primary care had declined to $28 \%$ while $50 \%$ of the NP workforce reported a primary care specialty (Figure 1).

PA specialty changes, 1997-2013 (Figure 2). The proportion of the PA workforce practicing in general primary care decreased from $43 \%$ in 1997 to just under $25 \%$ in 2013. Over the same period, the percentage of PAs reporting practice in adult medical specialties increased from $17 \%$ to $26 \%$. Ten percent of PAs practiced in urgent care in 2013, a specialty that was not included as a specialty option in either 1997 or 2005.

NP specialty changes, 1997-2013 (Figure 3). General primary care-general internal medicine, family medicine, and geriatrics-was the most common area of practice reported by NPs in all 3 years, with more than $40 \%$ reporting they worked in general primary care in 1997 and 2013 and slightly more than $50 \%$ working in this area in 2005 . The proportion reporting obstetrics/gynecology practice dropped from $21 \%$ to 5\% between 1997 and 2013, and the proportion in pediatric primary and specialty care also decreased. The largest area of growth was in adult medical subspecialties, where the proportion of NPs doubled from $12 \%$ in 1997 to $25 \%$ in 2013 . The proportion of NPs practicing in the neonatal/perinatal subspecialty decreased from $10 \%$ in 1997 to $4 \%$ in 2013 (data not shown).

- PAs and NPs practice in different specialties (Figure 4). In 2013, NPs were much more likely to practice in general primary care than PAs, with $43 \%$ of the NP workforce and $25 \%$ of the PA workforce reporting they worked in general internal medicine, family medicine, or geriatrics.
PAs and NPs were equally likely to be found in adult medical specialties but NPs were more likely to report practicing in general and specialty pediatrics as well as obstetrics/gynecology. A larger proportion of PAs reported working in procedural specialties such as surgery and emergency medicine. A relatively large proportion of PAs $(10 \%)$ in 2013 reported working in urgent care, but this specialty choice was not on the list of specialties offered to NPs in any year or PAs before 2013.

A more granular look at specific subspecialties in 2013 reveals additional differences. NPs are more likely to report practice in psychiatry, obstetrics/gynecology, and neonatal/ perinatal care, and are somewhat more likely to report practice in cardiology, compared with PAs. PAs are more likely to report practice in surgical and emergency/urgent areas of care, as well as dermatology and gastroenterology (Figure 5).

\section{DISCUSSION}

North Carolina, like other states and the nation, has experienced a rapid growth of PAs and NPs relative to physicians and the population over the past 15 years. ${ }^{14}$ The specialty distribution of both PAs and NPs changed over time and different specialty patterns emerged between the two professions. The percentage of the NP workforce in general primary care remained over $40 \%$ between 1997 and 2013, while the percentage of PAs in general primary care declined from $43 \%$ in 2005 to $25 \%$ in 2013. The relatively large proportion of PAs reporting practice in urgent care in $2013(10 \%)$ could partly explain the significant drop in PAs reporting a primary

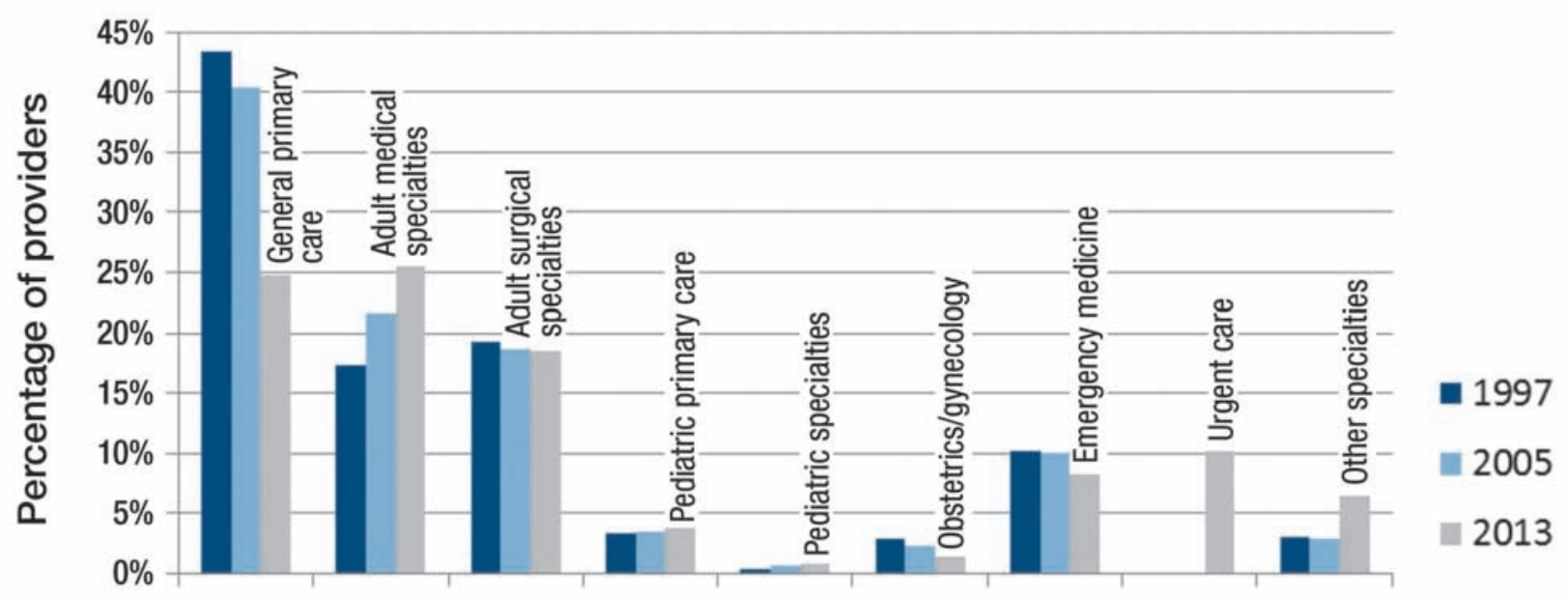

FIGURE 2. Percentage of active PAs by specialty categories in North Carolina in 1997, 2005, and 2013. General primary care is defined as general internal medicine, family medicine, and geriatrics. Pediatric primary care (including adolescent medicine) is a separate category. Providers reporting a specialty of hospitalist are grouped in adult medical specialties. 


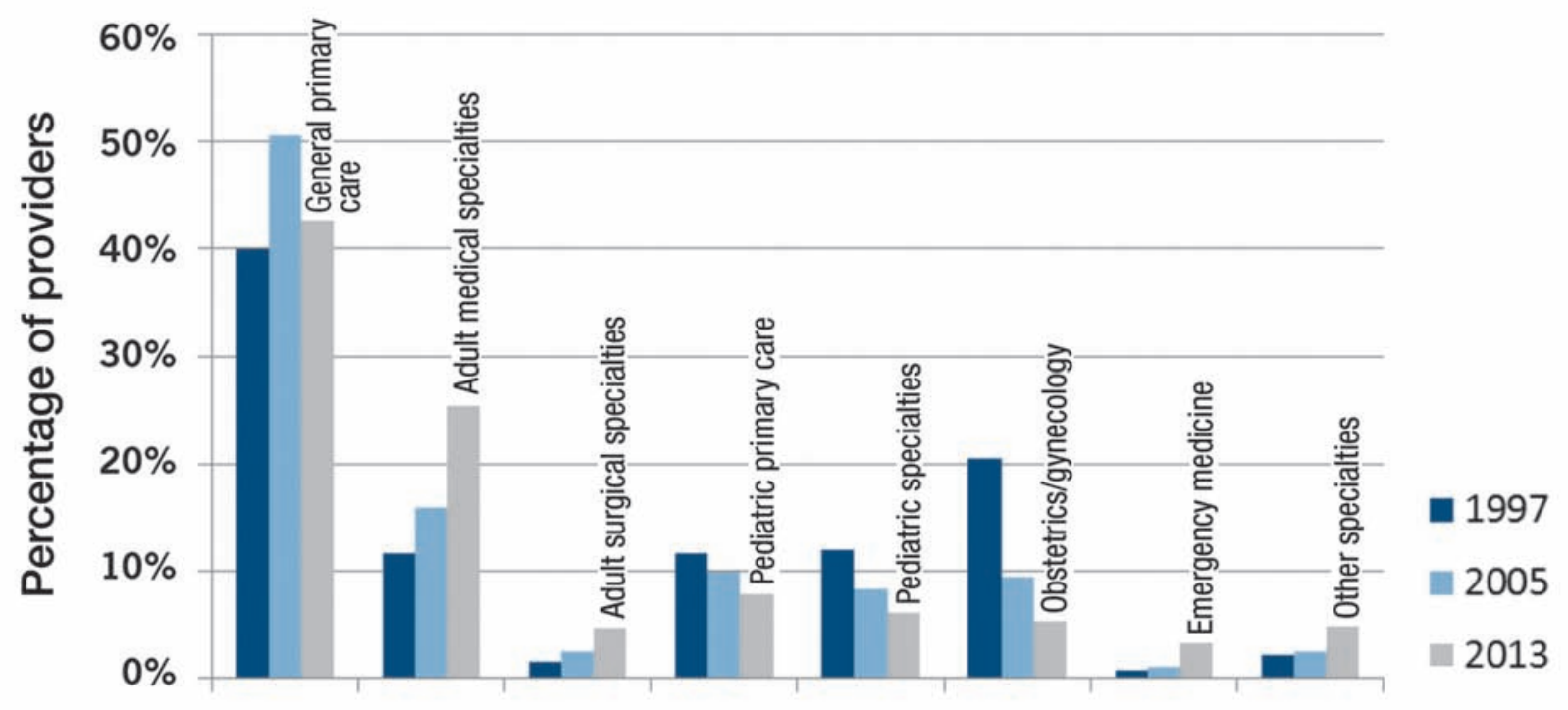

FIGURE 3. Percentage of active NPs by specialty categories in North Carolina in 1997, 2005, and 2013. General primary care is defined as general internal medicine, family medicine, and geriatrics. Pediatric primary care (including adolescent medicine) is a separate category. Providers reporting a specialty of hospitalist are grouped in adult medical specialties.

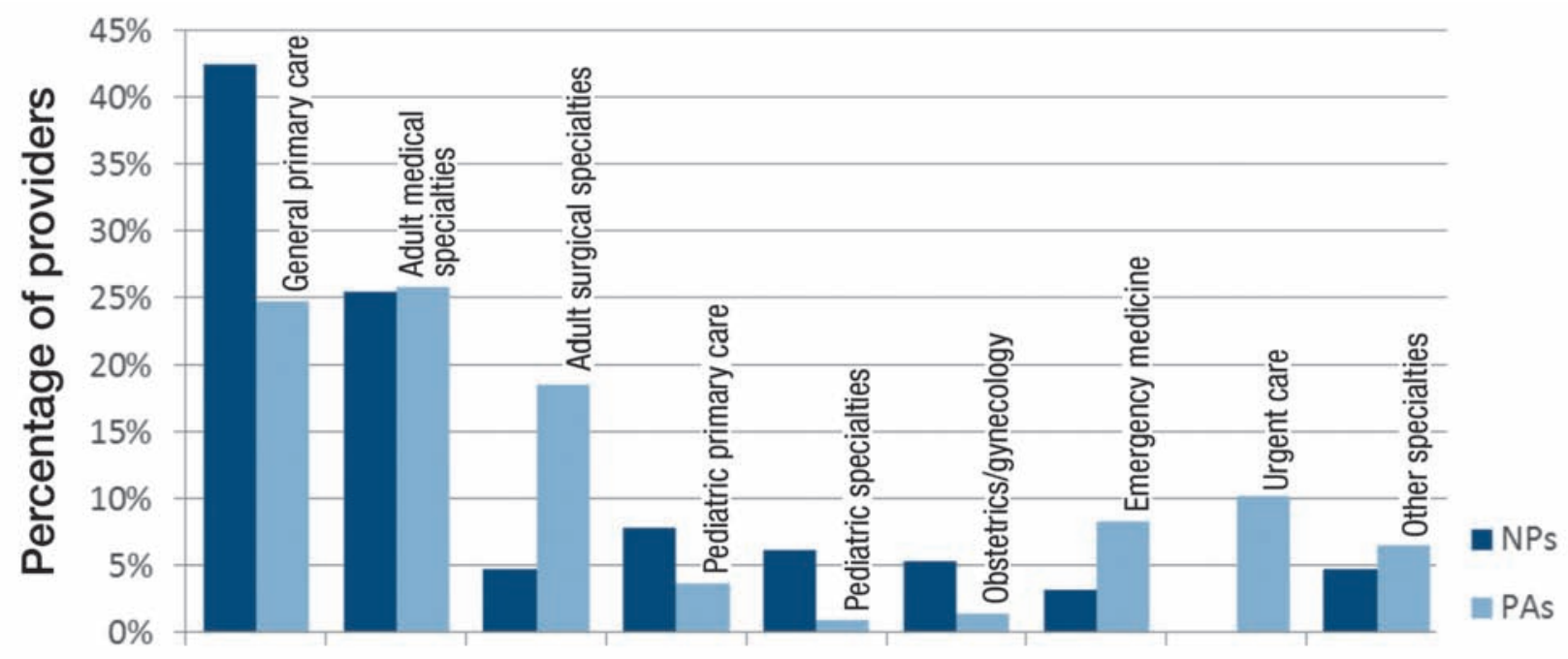

FIGURE 4. Percentage of active PAs and NPs by specialty categories in North Carolina in 2013. General primary care is defined as general internal medicine, family medicine, and geriatrics. Pediatric primary care (including adolescent medicine) is a separate category.

care specialty. Before 2013, when urgent care was added as a distinct specialty category, PAs who practiced in urgent care centers likely reported their specialty as either primary care or emergency medicine. The addition of this category may have falsely exaggerated the per- centage decline of PAs in general primary care and/or emergency medicine. Our study cannot discern the degree to which the proportion of PAs in primary care in 2013 reflects a correction of previously inaccurate categorization as opposed to actual movement of PAs into urgent 
care practice. Conversely, the lack of an urgent care specialty category for NPs could lead to overestimation of the general primary care or emergency medicine proportion of NPs.

Both PAs and NPs were more likely to practice in adult medical specialties in 2013 than they were in 1997, possibly reflecting the subspecialization of healthcare in response to the growing demand for specialty care from an aging population with an increased burden of chronic disease. ${ }^{18}$ Although NPs remain more likely than PAs to practice in obstetrics/gynecology and pediatric specialties than PAs, NPs' representation in these fields has declined, particularly in obstetrics/gynecology. The relatively smaller proportion of both PAs and NPs practicing in obstetrics/gynecology in recent years may reflect declining birth rates and/or regulatory and insurance barriers that discouraged PA and NP participation in labor and delivery services. ${ }^{19}$

North Carolina reflects national trends, with more PAs than NPs in procedural and surgical specialties. ${ }^{14,15}$ As Morgan and colleagues (in press) have suggested, surgeons may increase surgical volume and total revenue by shifting preoperative and postoperative tasks to PAs. ${ }^{14}$ This would provide an appealing business model promoting uptake of PAs in surgical specialties. In North Carolina, this trend is particularly striking in orthopedic surgery.

\section{LIMITATIONS}

This study has a number of important limitations. Although the PA and NP workforce in North Carolina generally mirrors the national workforce in terms of age, sex, and specialty distribution, important differences in the regulation of PAs and NPs may affect their deployment. North Carolina requires physician supervision for NPs and thus has a more restrictive scope of practice for NPs relative to other states. North Carolina has a less restrictive scope of practice for PAs relative to other states.

Another important limitation is that a significant percentage of NPs were missing specialty data: $16 \%$ of NPs did not report a specialty in $1997,15 \%$ in 2005 , and $34 \%$ in 2013 . However, with regard to age, race/ethnicity, and graduation year, NPs who were missing a specialty were not statistically significantly different from NPs who reported a specialty.

\section{IMPLICATIONS FOR WORKFORCE MODELING}

To account for the changing and differing specialty distributions of PAs and NPs over time, workforce modelers need more information about the factors that affect the demand and supply of PAs and NPs in different specialties. Supply-side factors include how a provider's age, race, sex, and preferences affect specialty choice. Systemlevel factors such as salary differentials between specialties, loan forgiveness for some specialties and not others, and the different training emphases of PAs and NPs affect how new graduates choose a specialty and why PAs and NPs already in the workforce may change specialties. On the demand side, we need a better understanding of the factors that induce organizations to hire PAs and NPs and how the growth in use of different types of healthcare services may differentially affect the demand for PAs versus NPs.

This analysis suggests that despite considerable overlap among PA and NP specialties, these providers are not an interchangeable resource. If the demand for surgical and procedural specialties increases in the future,

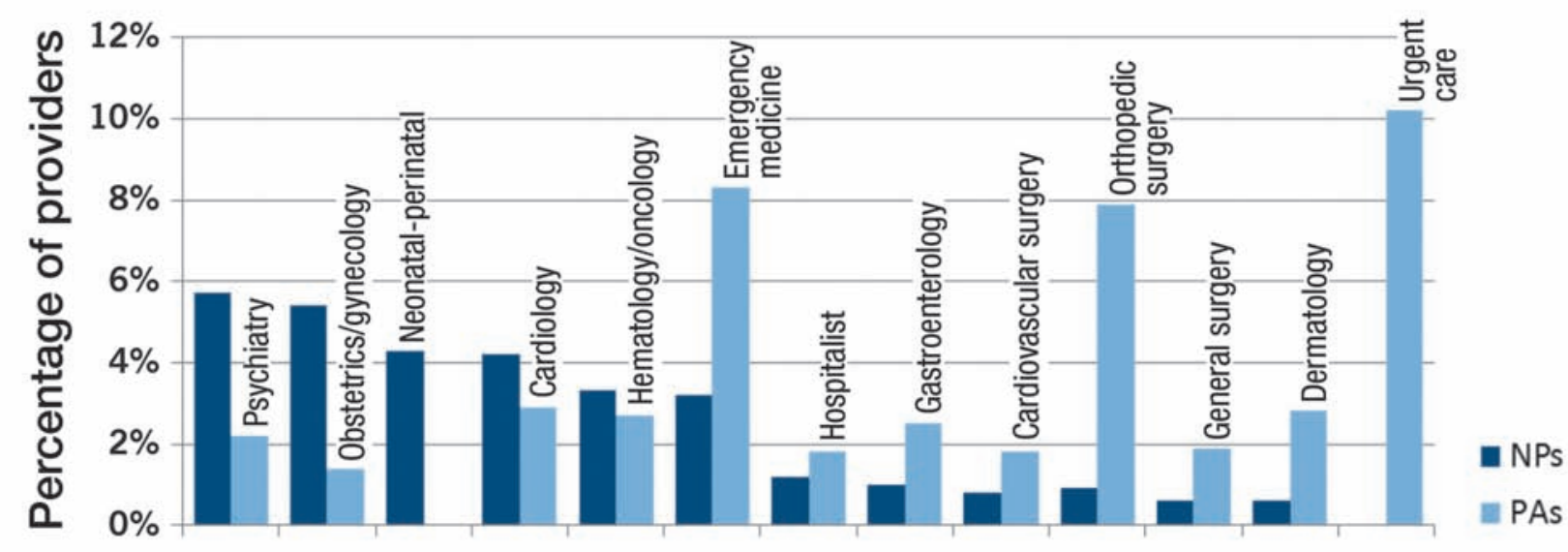

FIGURE 5. Percentage of active NPs by specialty categories in North Carolina in 1997, 2005, and 2013. Urgent care was not available for NPs to select. 
the uptake of PAs into these specialties will likely increase at a faster rate than for NPs. By contrast, if new models of care such as patient-centered medical homes and accountable care organizations continue to be adopted, this may increase the demand for NPs relative to PAs because NPs are more likely to practice in preventive and primary care.

The specialty distribution of PAs and NPs also is likely to change over time. As payment models move toward risk- and value-based systems, healthcare employers will increasingly look to shift work toward PAs and NPs, whose salaries are one-third to one-half those of physicians. ${ }^{14,20}$ This trend may increase the numbers of PAs and NPs employed in all specialties, but especially in subspecialties where the salary differentials between physicians and PAs and NPs are greatest. However, two important constraints exist on the substitution rate of PAs and NPs for physicians in different specialties-physician acceptance of PAs and NPs and the degree of potential task substitution in the specialty.

The lack of data on both the supply and demand factors that affect PA and NP specialty distribution has hindered the development of interdisciplinary workforce models that include physicians, PAs, and NPs. The latest AAMC model did not include a scenario incorporating the growth of PAs, citing a lack of available data to quantify the implication of increasing PA-to-physician staffing ratios. ${ }^{1,21}$ The latest HRSA model included a scenario showing that increased deployment of PAs and NPs in primary care reduced the estimated shortfall in 2020 from 20,400 to only 6,400 primary care providers. The acknowledgment in both models of the large effect that PA and NP supply can have on physician workforce projections is an important step forward for the field. The next step is to develop scenarios that treat PAs and NPs differently in terms of their specialty distribution and to model how their specialty distributions might change over time in response to individual and system level supply and demand factors. JAAPA

\section{REFERENCES}

1. Association of American Medical Colleges. The Complexities of Physician Supply and Demand: Projections from 2013 to 2025. https://www.aamc.org/download/426242/data/ihsreportdownload.pdf. Accessed January 12, 2016.

2. US Department of Health and Human Services, Health Resources and Services Administration, Bureau of Health. Projecting the Supply and Demand for Primary Care Practitioners through 2020. http://bhpr.hrsa.gov/healthworkforce/ supplydemand/usworkforce/primarycare/projectingprimarycare. pdf. Accessed January 12, 2016.

3. Glied S, Ma S. How will the Affordable Care Act affect the use of health care services? The Commonwealth Fund. www. commonwealthfund.org/publications/issue-briefs/2015/feb/ how-will-aca-affect-use-health-services. Accessed January 12, 2016.

4. Fraher E. A Primer on the Health Care Workforce in the United States [presentation0]. National Health Policy Forum,
Washington, DC. February 20, 2015. www.nhpf.org/uploads/ Handouts/Fraher1-slides_02-20-15.pdf. Accessed January 12, 2016.

5. Green LV, Savin S, Lu Y. Primary care physician shortages could be eliminated through use of teams, nonphysicians, and electronic communication. Health Aff (Millwood). 2013; 32(1):11-19.

6. Auerbach DI, Chen PG, Friedberg MW, et al. Nurse-managed health centers and patient-centered medical homes could mitigate expected primary care physician shortage. Health Aff (Millwood). 2013;32(11):1933-1941.

7. Dill MJ, Pankow S, Erikson C, Shipman S. Survey shows consumers open to a greater role for physician assistants and nurse practitioners. Health Aff (Millwood). 2013;32(6):1135-1142.

8. Hooker RS, Cawley JF, Leinweber W. Career flexibility of physician assistants and the potential for more primary care. Health Aff (Millwood). 2010;29(5):880-886.

9. National Commission on Certification of Physician Assistants. Statistical Profile of Certified Physician Assistants. 2013. www. nccpa.net/Upload/PDFs/2013StatisticalProfileofCertifiedPhysician Assistants-AnAnnualReportoftheNCCPA.pdf. Accessed January $12,2016$.

10. US Department of Health and Human Services, Health Resources and Services Administration, National Center for Health Workforce Analysis. Highlights from the 2012 National Sample Survey of Nurse Practitioners. http://bhpr.hrsa.gov/ healthworkforce/supplydemand/nursing/nursepractitionersurvey/ npsurveyhighlights.pdf. Accessed January 27, 2016.

11. Hooker RS, Berlin LE. Trends in the supply of physician assistants and nurse practitioners in the United States. Health Aff (Millwood). 2002;21(5):174-181.

12. Hopkins SC, Lenz ER, Pontes NM, et al. Context of care or provider training: the impact on preventive screening practices. Prev Med. 2005;40(6):718-724.

13. Mills AC, McSweeney M. Nurse practitioners and physician assistants revisited: do their practice patterns differ in ambulatory care? J Prof Nurs. 2002;18(1):36-46.

14. Morgan P, Everett C, Humeniuk K, Valentin VL. Specialty choice among US physician assistants: distribution, salaries, and comparison to physicians. JAAPA. In press.

15. Hooker RS, Brock DM, Cook ML. Characteristics of nurse practitioners and physician assistants in the United States. J Am Assoc Nurse Pract. 2016;28(1):39-46.

16. American Association of Nurse Practitioners. State Practice Environment. 2014. www.aanp.org/legislation-regulation/ state-legislation-regulation/state-practice-environment/66legislation-regulation/state-practice-environment/1380-statepractice-by-type. Accessed January 12, 2016.

17. Agency for Healthcare Research and Quality. The Number of Nurse Practitioners and Physician Assistants Practicing Primary Care in the United States: Primary Care Workforce Facts and Stats No. 2. 2014. www.ahrq.gov/research/findings/factsheets/ primary/pcwork2/index.html. Accessed January 12, 2016.

18. Dall TM, Gallo PD, Chakrabarti R, et al. An aging population and growing disease burden will require a large and specialized health care workforce by 2025. Health Aff (Millwood). 2013; 32(11):2013-2020.

19. Martin JA, Hamilton BE, Osterman JK, et al. Births: Final Data for 2012. National Vital Statistics Reports, National Center for Health Statistics, Hyattsville, MD, 2013.

20. Medical Group Management Association. MGMA Physician Compensation and Production Survey. 2012. www.mgma. com/industry-data/mgma-surveys-reports. Accessed January $27,2016$.

21. Salsberg ES. Is the physician shortage real? Implications for the recommendations of the Institute of Medicine Committee on the Governance and Financing of Graduate Medical Education. Acad Med. 2015;90(9):1210-1214. 